\title{
Zs in teens: Impact of parental pressure on adolescent sleep patterns
}

\section{S Yamuna}

Child and Adolescent Clinic, Thiruvanmiyur, Chennai

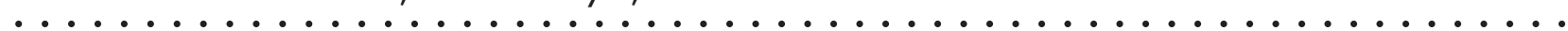

Indian J Sleep Med 2009; 4.2, 45-48

\begin{abstract}
Adolescents are marching towards independence with the guidance of parents for a decade. Parents who are usually in their fifth decades tend to expect adolescents to be mature enough to behave like themselves. This places a major demand on the adolescents who are struggling to manage their own physiological shifts and emotional upheavals of the period. Parenting styles, misconceptions, ignorance and anxiety have a significant role to play in altering the sleep patterns of the adolescents. Hence it is mandatory that health care providers should be equipped with the changes expected in sleep patterns during adolescence and must be empowered with skills to convince parents to expect and accept the same.
\end{abstract}

A dolescents are on a journey towards adulthood but under the constant supervision and guidance or misguidance by many adults around them. Parents play a major role in supporting the growth and development of adolescents as the roof is shared by almost all Indian adolescents with their parents unless there is a migration due to education or employment.

Adolescence is itself seen as a new phase of development that is focused on in view of the attachment to more number of years of formal education that has been gaining popularity in recent times. More number of years of formal education has been associated with better job prospects with less of mechanical exertion. Longer period of education increases the financial dependence on parents and caretakers and thus adolescents in spite of their individual needs and decision making skills are constantly under supervision and

\section{Address for correspondence}

\section{Dr S Yamuna}

Consultant Paediatrician and Adolescent Physician, Child and Adolescent Clinic

H 110/ S 2, Waves, Third Seaward Road, Valmiki Nagar, Thiruvanmiyur, Chennai 600041.

Ph 04424426535 Cell 9381007928

E mail: dryamunapaed@yahoo.com monitoring with critical as well as enlightening inputs from parents who are their providers.

Puberty as such brings on numerous changes in the metabolic, phenotypic, emotional as well as perceptual domains of a person. Puberty in a boy or a girl is also associated with many modifications in the expectations of many adults around them. Parental expectations are related to what they practiced or achieved during their adolescence when the technical advancement was way behind than the current days.

Puberty also has a significant impact on the sleep pattern and sleep needs of an adolescent. This article is aimed at highlighting the physiological changes that happen during adolescence with respect to sleep needs and sleep patterns and how this is being modified by influences from parents.

India being a country with development in multiple domains is seeing growth of the cities to a large extent. Peer pressure among parents makes the parents resort to practices in their adolescents without considering the consequences. Hence it becomes the duty of the primary health care providers to probe into the sleeping practices of the family and the children for identifying abnormal influences at an early stage of development so that behavior modification is feasible for the betterment of the individual under consideration. In the presence of abnormal sleep patterns, 
sleep specialists can be approached for appropriate interventions.

\section{Sleep changes during adolescence}

\section{Adolescents need at least 9 hours of sleep every 24 hours of wakefulness}

The circadian rhythm of a person is slightly more than 24 hours. Restful sleep of specified duration is necessary for recuperation and restoration of functions of all the systems of the body. Normative data suggest that adolescents should sleep at least 9 hours every 24 hour cycle to feel rested and rejuvenated. Do they get this duration of uninterrupted sleep? Their activities demand more wakeful hours and thus adolescents are walking around with a sleep debt that is usually not fulfilled. Hence their sleep demand is high and they appear sleepy or drowsy during hours when they cannot sleep due to social expectations as in a classroom setting.

\section{Two hours shift in the circadian sleep phase}

During adolescence there is phase shift of two hours. If a boy or a girl is used to sleeping by 9 pm every night, after puberty their sleeping time is usually pushed to $11 \mathrm{pm}$ even in the absence of environmental alterations. The adolescent will not be able to fall asleep for two hours even if they are on bed. This is because of changes in the secretion of hormones like melatonin and sex steroids. This shift keeps them awake until late and this should be communicated to parents, who, find the change very annoying.

\section{Difficulty to wake up early}

As the onset of sleep is delayed the waking time is also pushed to late morning. Hence adolescents find waking up to school very troublesome and most of the adolescents look very sleepy during the morning classes. This sleepiness has major impact on functions of the adolescents with respect to maintenance of personal hygiene and food intake. Recommendations are being made to make the school timings of middle and high school later than that of the younger children.

\section{Sleep latency falls in the afternoon}

During adolescence the sleep latency, i.e the time in bed before onset of sleep shortens in the afternoon. The adolescent who sleeps late but wakes up early either with an alarm or parents' call, have a very high sleep debt.
Thus the ease with which an adolescent can fall asleep during afternoon classes is very high especially if the teacher has a monotonous voice or if they are involved in passive listening.

\section{Dead zone/ Forbidden zone in the evenings}

All human beings have a forbidden zone during which the person is very alert and is incapable of sleeping as they remain alert to environment. This zone which was all along in the afternoon in smaller children gets shifted to evenings. During the forbidden zone of the day adolescents are usually resistant to sleep.

\section{Sleep deprived as the activities demand more wakeful hours}

Adolescents are active on the fields due to sports; the academic demands make them study for long hours. Coaching classes, tuition and remedial classes increase the working hours. Hence adolescents sacrifice their sleep to catch up with their socialization, recreation and leisure activities.

\section{Sleep debt impairs memory, behavior, mood, attention, scholastic performance}

A person should have the required number of hours for sleeping to restore the functions of the brain. In the absence of restful sleep, restlessness is manifested as irritability, anger, forgetfulness, lack of attention and concentration, with fall in school grades.

\section{Drowsiness makes them prone for accidents}

Sleep deprivation makes the adolescents have a very low threshold to sleep while they are awake and this makes them close their eyes if the scenes are monotonous. This has contributed to many road traffic accidents as the adolescents would lose control over the wheel or the cycle.

\section{Parental Expectations}

Parents of both genders of current days are committed to employment in addition to child care and family affairs. This gives them less time to interact with their children and this happens with adolescents as adolescents are within their shells if left unattended. Lack of communication on issues of interest makes the drift between parents and adolescents 
get wider. Parents tend to look at adolescents as the mature lot who should be always responsible and studious as it is their duty to study and progress. Hence a lot of pressure is placed on their performance and not on their maintenance.

Conversations are limited to academics, assessments, achievements and accomplishments as a result other areas are often neglected. Adolescents are given an opinion that the only way they can see a smile on parents' faces is when they can show a good report card. Hence many adolescents force themselves in having a selfimposed target to make their parents' approve of them as good boys and girls. This makes them get used to erratic sleep rhythm that does not help the adolescents have a restorative sleep.

Secondly, the parenting styles contribute a lot towards the sleep calendar.

Authoritarian parents who believe in "Do what I say" force their children and adolescents to follow a pattern that suits the parents during their fifth decade forgetting that the adolescents are only in their second decade of life. Adolescents are forced to focus on curriculum and extra curricular activities when the circadian sleep cycle is not ready for the activities. This makes the adolescents fail in their acquisition of skills and the outcome being that the parents force the adolescents to try further at the same appointed hour. The role of health care providers is to help parents understand that the sleep needs and sleep patterns of the second decade are much different from that of the fifth decade.

The Permissive parents, feel that the adolescents are grown ups and they deserve all comforts in life. They go ahead in providing a personal computer with a broad band connection, I pod, cell phone, television with satellite connection, DVD player etc for their adolescents and allow the children to have all these devices inside the bedrooms without realising that these gadgets would make the bedroom very busy during the silent sleepy hours of the night. There are a few parents who believe that having independent sets of gadgets helps in maintaining viewing freedom and believe that adolescents and the parents can choose to have the entertainment of their choice within the confines of their bedrooms without disturbing the other party.

The Negligent parents tend to believe that their role in rearing the child has come to an end the moment he or she is capable of looking after self care needs. They give a set of keys to the adolescents and each member of the family has a different time of return without any common meals or curfew times. These are the parents who believe that substance use, experimentation with sexual activities as rite to passage into adulthood and there are no questions asked about the daily routine of their sons, but less so with their daughters. They intervene only when there are crises and generally leave the adolescents to learn by themselves from their various activities. Late evenings with frequent sleep overs are common among these adolescents with a very chaotic sleep calendar.

Over indulgent or over anxious parents are another extreme who do not let their adolescents develop decision making skills as they feel that the adolescents will have to depend on them until they are financially dependent on the parents. Fear of retirement from parenting makes them get over involved in the adolescent care to the extent they would be aware of the adolescent's last visit to the toilet before bedtime and the position in which he or she sleeps most of the nights.

Some parents have rigid parenting for six days a week with a relaxed day on Sundays when no questions are asked. This makes the adolescents have a long sleep sometimes till $2 \mathrm{pm}$ on Sundays that the sleep rhythm is altered with consequent sleep irregularities.

Co sleeping parents believe that the adolescents have to be with them as they would lose touch with their sons and daughters when they get married or when they migrate for education or employment. They go to the extent of moving out of their bedrooms to a common place where the entire family can be accommodated on the floor. Co sleeping is a common practice among resource poor Indian families in view of lack of separate spaces for each member of the family. But recently we observe a trend where even in the presence of exclusive spaces for each of the family members, co sleeping and bed sharing are practiced for reasons known only to the parents. Some parents believe that monitoring the adolescents' activities is possible only by co sleeping with them.

Environmental factors play a major role in deciding the sleep patterns of the children as the environment has a significant impact on the psychosocial milieu of the adolescent. Emotional divorces, parental substance abuse, domestic violence, marital disharmony, extramarital affairs, are common in recent times. Adolescents are prone to be eye witnesses to the whole set of events. Also adolescents have a better set of antennae to detect disquiet between parents even in the absence of expressed displeasure by parents. These emotional upheavals leave an indelible mark in the psychosocial well being of the adolescents and are

Indian Journal of Sleep Medicine (IJSM), Vol. 4, No. 2, 2009 
usually manifested as altered sleep rhythms or parasomnias to a certain extent.

Rights of adolescents are commonly forgotten by parents. Right to privacy especially with respect to conversations with peer group is something every adolescent is entitled to. The parents tend to forget that their sons and daughters interact with their peer group for almost eight hours a day at schools without the parental supervision. Many parents insist that their adolescents can converse with their friends only in their presence. This annoys many adolescents and many of them instruct their friends to wait until the parents fall asleep sacrificing their own sleep needs, thus extending the wakeful hours with inadequate sleep every day with weekend compensation.
Parental expectations and limit settings are dependent on many myths and misconceptions that prevail in our community for generations now. We as health care providers have a huge role to play in busting these myths and in providing appropriate tips towards maintenance of sleep hygiene. Adolescents are under pressure to manage their parents, their own physiological changes, academic demands, self imposed targets and many other media inputs and thus are immensely in need of help to arrive at a sleep pattern that is likely to help them achieve most of their objectives. 